\title{
RATIONAL FUNCTION VARIANT OF A PROBLEM OF ERDÖS AND GRAHAM
}

\author{
Szabolcs Tengely AND Nóra VARga
}

Hungarian Academy of Sciences and University of Debrecen, Hungary

ABSTRACT. In this paper we provide bounds for the size of the solutions of the Diophantine equations

$$
\begin{aligned}
& \frac{x(x+1)(x+2)(x+3)(x+4)(x+5)}{(x+a)(x+b)}=y^{2}, \\
& \frac{x(x+1)(x+2)(x+3)(x+4)(x+5)}{(x+a)(x+b)(x+c)}=y^{3}, \\
& \frac{x(x+1)(x+2)(x+3)(x+4)(x+5)}{(x+a)(x+b)(x+c)(x+d)}=y^{2},
\end{aligned}
$$

where $a, b, c, d \in \mathbb{Z}$ are pairwise distinct integers.

\section{INTRODUCTION}

Let us define

$$
f(x, k, d)=x(x+d) \cdots(x+(k-1) d),
$$

and consider the Diophantine equation

$$
f(x, k, d)=y^{l} .
$$

Erdős ([5]) and independently Rigge ([17]) proved that the equation $f(x, k, 1)=y^{2}$ has no integer solution. Erdős and Selfridge ([7]) extended this result when $d=1, x \geq 1$ and $k \geq 2$ and they stated that $f(x, k, 1)$ is

2010 Mathematics Subject Classification. 11D61, 11Y50.

Key words and phrases. Diophantine equations.

Szabolcs Tengely was supported by the European Union and the State of Hungary, co-financed by the European Social Fund in the framework of TÁMOP 4.2.4. A/2-111-2012-0001 "National Excellence Program". Nóra Varga was supported by the European Union and the State of Hungary, co-financed by the European Social Fund in the framework of TÁMOP 4.2.4. A/2-11-1-2012-0001 "National Excellence Program". 
never a perfect power. This type of Diophantine equations have been studied intensively.

First assume that $l=2$. Euler solved the equation (1.1) with $k=4$ (see [4, pp. 440 and 635]) and after that Obláth ([16]) extended this result to the product of five terms in arithmetic progression, i.e. $k=5$. If $d$ is a power of a prime number and $k \geq 4$ Saradha and Shorey ([20]) proved that (1.1) has no solutions. Laishram and Shorey $([14])$ examined the case where either $d \leq 10^{10}$, or $d$ has at most six prime divisors. Bennett, Bruin, Györy and Hajdu ([2]) solved (1.1) when $6 \leq k \leq 11$. Hirata-Kohno, Laishram, Shorey and Tijdeman ([13]) completely solved the equation (1.1) with $3 \leq k<110$. Combining their result with those of Tengely ([23]) all solutions of (1.1) with $3 \leq k \leq 100, P(b)<k$ are determined, where $P(u)$ denotes the greatest prime factor of $u$, with the convention $P(1)=1$.

Now assume for this paragraph that $l \geq 3$. The literature of this equation

$$
f(x, k, d)=b y^{l},
$$

with $b>0$ and $P(b) \leq k$ is very rich. Saradha ([19]) proved that (1.2) has no solution with $k \geq 4$. Györy ([9]) studied the product of two and three consecutive terms in arithmetic progression. Győry, Hajdu and Saradha $([11])$ proved that if $k=4,5$ and $\operatorname{gcd}(x, d)=1$ equation (1.2) cannot be a perfect power. Hajdu, Tengely and Tijdeman ([12]) proved that the product of $k$ coprime integers in arithmetic progression cannot be a cube when $2<k<39$. Bennett, Bruin, Győry and Hajdu ([2]) gave various finiteness results under the assumption that $k$ is fixed. If $3<k<35$ and $\operatorname{gcd}(x, d)=1$ Györy, Hajdu and Pintér ([10]) proved that for any positive integers $x, d$ and $k$ the product $f(x, k, d)$ cannot be a perfect power.

Erdős and Graham ([6]) asked if for fixed $r \geq 1$ and $\left\{k_{1}, k_{2}, \ldots, k_{r}\right\}$ with $k_{i} \geq 4$ for $i=1,2, \ldots, r$ the Diophantine equation

$$
\prod_{i=1}^{r} f\left(x_{i}, k_{i}, 1\right)=y^{2}
$$

has at most finitely many solutions in positive integers $\left(x_{1}, x_{2}, \ldots, x_{r}, y\right)$ with $x_{i}+k_{i} \leq x_{i+1}$ for $1 \leq i \leq r-1$. Skatba ([21]) provided a bound for the smallest solution and estimated the number of solutions below a given bound. Ulas ([25]) gave a counterexample when either $r=k_{i}=4$, or $r \geq 6$ and $k_{i}=4$. Bauer and Bennett ([1]) extended this result to the cases $r=3$ and $r=5$. In the case $k_{i}=5$ and $r \geq 5$ Bennett and Van Luijk ([3]) constructed an infinite family such that the product $\prod_{i=1}^{r} f\left(x_{i}, k_{i}, 1\right)$ is always a perfect square. Luca and Walsh ([15]) considered the case $\left(r, k_{i}\right)=(2,4)$.

In our previous paper [24] we considered the equation

$$
\frac{x(x+1)(x+2)(x+3)}{(x+a)(x+b)}=y^{2}
$$


where $a, b \in \mathbb{Z}, a \neq b$ are parameters. We provided bounds for the size of solutions and an algorithm to determine all solutions $(x, y) \in \mathbb{Z}^{2}$. The proof based on Runge's method and the result of Sankaranarayanan and Saradha ([18]).

In this paper we extend this latter result and study the following three Diophantine equations

$$
\begin{aligned}
& \frac{x(x+1)(x+2)(x+3)(x+4)(x+5)}{(x+a)(x+b)}=y^{2}, \\
& \frac{x(x+1)(x+2)(x+3)(x+4)(x+5)}{(x+a)(x+b)(x+c)}=y^{3}, \\
& \frac{x(x+1)(x+2)(x+3)(x+4)(x+5)}{(x+a)(x+b)(x+c)(x+d)}=y^{2},
\end{aligned}
$$

where $a, b, c, d \in \mathbb{Z}$ are pairwise distinct integers such that $a, b, c, d \notin$ $\{0,1,2,3,4,5\}$. Bounds for the solutions of these equations are provided in the following three theorems.

Theorem 1.1. If $(x, y) \in \mathbb{Z}^{2}$ is a solution of the Diophantine equation

$$
\frac{x(x+1)(x+2)(x+3)(x+4)(x+5)}{(x+a)(x+b)}=y^{2},
$$

then either

$$
x \mid\left(3 a^{2}+2 a b+3 b^{2}-30 a-30 b+115\right)^{2} a b
$$

or

$$
|x| \leq 16 t^{3}+440 t^{2}
$$

where $a, b \notin\{0,1,2,3,4,5\}$ and $t=\max \{|a|,|b|\}$.

Theorem 1.2. If $(x, y) \in \mathbb{Z}^{2}$ is a solution of the Diophantine equation

$$
\frac{x(x+1)(x+2)(x+3)(x+4)(x+5)}{(x+a)(x+b)(x+c)}=y^{3},
$$

then either

$$
x \mid(a+b+c-15)^{3} a b c
$$

or

$$
|x| \leq 6 t^{2}+68 t
$$

where $a, b, c \notin\{0,1,2,3,4,5\}$ and $t=\max \{|a|,|b|,|c|\}$.

Theorem 1.3. If $(x, y) \in \mathbb{Z}^{2}$ is a solution of the Diophantine equation

$$
\frac{x(x+1)(x+2)(x+3)(x+4)(x+5)}{(x+a)(x+b)(x+c)(x+d)}=y^{2},
$$

then either

$$
x \mid(a+b+c+d-15)^{2} a b c d
$$


or

$$
|x| \leq 12 t^{2}+132 t
$$

where $a, b, c, d \notin\{0,1,2,3,4,5\}$ and $t=\max \{|a|,|b|,|c|,|d|\}$.

We will use the following result of Fujiwara ([8]) to prove our statements.

Lemma 1.4. Let $p(z)=\sum_{i=0}^{n} a_{i} z^{i}$ be a polynomial in $\mathbb{C}[z]$ with $a_{n} \neq 0$. Then

$$
\max \{|\zeta|: p(\zeta)=0\} \leq 2 \max \left\{\left|\frac{a_{n-1}}{a_{n}}\right|,\left|\frac{a_{n-2}}{a_{n}}\right|^{1 / 2}, \ldots,\left|\frac{a_{0}}{a_{n}}\right|^{1 / n}\right\} .
$$

\section{Proof of Theorem 1}

We deal with the equation

$$
F(x)=\frac{x(x+1)(x+2)(x+3)(x+4)(x+5)}{(x+a)(x+b)}=y^{2} .
$$

The polynomial part of the Puiseux expansion of $F(x)^{1 / 2}$ is

$$
P(x)=x^{2}-\frac{a+b-15}{2} x+\frac{3 a^{2}+2 a b+3 b^{2}-30 a-30 b+115}{8} .
$$

Let

$$
A(x)=x(x+1)(x+2)(x+3)(x+4)(x+5)-(x+a)(x+b)\left(P(x)-\frac{1}{8}\right)^{2}
$$

and

$$
B(x)=x(x+1)(x+2)(x+3)(x+4)(x+5)-(x+a)(x+b)\left(P(x)+\frac{1}{8}\right)^{2}
$$

We have that $\operatorname{deg} A=\operatorname{deg} B=4$ and the leading coefficient of $A$ is $1 / 4$ and the leading coefficient of $B$ is $-1 / 4$. Denote by $I_{A}$ an interval containing all zeroes of the polynomial $A(x)$ and by $I_{B}$ the interval containing all zeroes of $B(x)$. We observe that if $x<\min \{a, b\}$ or $x>\max \{a, b\}$ and we also have that $x \notin I_{A}, x \notin I_{B}$, then

$$
\frac{A(x)}{(x+a)(x+b)} \text { and } \frac{B(x)}{(x+a)(x+b)}
$$

have opposite signs. Therefore there are two possibilities. Either

$$
\begin{aligned}
& F(x)-\left(P(x)-\frac{1}{8}\right)^{2}<0 \\
& F(x)-\left(P(x)+\frac{1}{8}\right)^{2}>0
\end{aligned}
$$


or

$$
\begin{aligned}
& F(x)-\left(P(x)-\frac{1}{8}\right)^{2}>0, \\
& F(x)-\left(P(x)+\frac{1}{8}\right)^{2}<0 .
\end{aligned}
$$

We only handle the first case, the second case is very similar. Here we obtain that

$$
\left(P(x)+\frac{1}{8}\right)^{2}<F(x)=y^{2}<\left(P(x)-\frac{1}{8}\right)^{2}
$$

Hence

$$
(8 P(x)+1)^{2}<(8 y)^{2}<(8 P(x)-1)^{2} .
$$

The polynomial $8 P(x)$ has integral coefficients, so if $x$ is an integer, then $8 P(x)$ is an integer as well. For a fixed integer $x$ there is only one square integer between $(8 P(x)+1)^{2}$ and $(8 P(x)-1)^{2}$, it is $64 P(x)^{2}$. Thus $y=P(x)$ and $x$ divides the constant term of the polynomial $64 x(x+1)(x+2)(x+3)(x+$ $4)(x+5)-64(x+a)(x+b) P(x)^{2}$, that is $x$ divides

$$
\left(3 a^{2}+2 a b+3 b^{2}-30 a-30 b+115\right)^{2} a b .
$$

It remains to provide an upper bound for the size of roots of $A(x)=\frac{1}{4} x^{4}+$ $a_{3} x^{3}+a_{2} x^{2}+a_{1} x+a_{0}$ and $B(x)=-\frac{1}{4} x^{4}+b_{3} x^{3}+b_{2} x^{2}+b_{1} x+b_{0}$. Let $t=\max \{|a|,|b|\}$. We have that

$$
\begin{aligned}
& \left|4 a_{3}\right| \leq 8 t^{3}+60 t^{2}+114 t+45 \\
& \left|4 a_{2}\right| \leq \frac{15}{4} t^{4}+60 t^{3}+450 t^{2}+855 t+\frac{1135}{4} \\
& \left|4 a_{1}\right| \leq \frac{9}{4} t^{5}+45 t^{4}+282 t^{3}+855 t^{2}+\frac{3249}{2} t+480 \\
& \left|4 a_{0}\right| \leq 4 t^{6}+60 t^{5}+339 t^{4}+855 t^{3}+\frac{3249}{4} t^{2} .
\end{aligned}
$$

Similarly we obtain that

$$
\begin{aligned}
& \left|4 b_{3}\right| \leq 8 t^{3}+60 t^{2}+116 t+30 \\
& \left|4 b_{2}\right| \leq \frac{15}{4} t^{4}+60 t^{3}+450 t^{2}+870 t+255 \\
& \left|4 b_{1}\right| \leq \frac{9}{4} t^{5}+45 t^{4}+283 t^{3}+870 t^{2}+1682 t+480 \\
& \left|4 b_{0}\right| \leq 4 t^{6}+60 t^{5}+341 t^{4}+870 t^{3}+841 t^{2}
\end{aligned}
$$

By Fujiwara's result it follows that

$$
\max \{|\zeta|: A(\zeta)=0 \text { or } B(\zeta)=0\} \leq 16 t^{3}+440 t^{2} .
$$




\section{Proof of Theorem 2}

Now, we consider the equation

$$
\frac{x(x+1)(x+2)(x+3)(x+4)(x+5)}{(x+a)(x+b)(x+c)}=y^{3},
$$

where $a, b, c \in \mathbb{Z}$ are pairwise distinct integers with $a, b, c \notin\{0,1,2,3,4,5\}$. The polynomial part of the Puiseux expansion of

$$
\left(\frac{x(x+1)(x+2)(x+3)(x+4)(x+5)}{(x+a)(x+b)(x+c)}\right)^{1 / 3}
$$

is $P(x)=x+5-\frac{a+b+c}{3}$. Define

$$
A(x)=x(x+1)(x+2)(x+3)(x+4)(x+5)-(x+a)(x+b)(x+c)\left(P(x)-\frac{1}{3}\right)^{3}
$$

and

$$
B(x)=x(x+1)(x+2)(x+3)(x+4)(x+5)-(x+a)(x+b)(x+c)\left(P(x)+\frac{1}{3}\right)^{3} .
$$

We obtain that $\operatorname{deg} A=\operatorname{deg} B=5$ and the leading coefficient of $A$ is 1 and the leading coefficient of $B$ is -1 . Therefore

$$
\frac{A(x)}{(x+a)(x+b)(x+c)} \text { and } \frac{B(x)}{(x+a)(x+b)(x+c)}
$$

have opposite signs if $|x|$ is larger than the maximum of the zeroes of $A(x) B(x)$ in absolute value. The following two possibilities can occur. Either

$$
\left(P(x)-\frac{1}{3}\right)^{3}<\frac{x(x+1)(x+2)(x+3)(x+4)(x+5)}{(x+a)(x+b)(x+c)}<\left(P(x)+\frac{1}{3}\right)^{3}
$$

or

$$
\left(P(x)+\frac{1}{3}\right)^{3}<\frac{x(x+1)(x+2)(x+3)(x+4)(x+5)}{(x+a)(x+b)(x+c)}<\left(P(x)-\frac{1}{3}\right)^{3} .
$$

In a similar way than in the proof of Theorem 1 one gets that $y=P(x)=$ $x+5-\frac{a+b+c}{3}$. Hence $x$ divides the constant coefficient of the polynomial

$$
27 x(x+1)(x+2)(x+3)(x+4)(x+5)-27(x+a)(x+b)(x+c) P(x)^{3},
$$

that is

$$
x \mid(a+b+c-15)^{3} a b c .
$$

It remains to determine a bound for the maximum of the zeroes of $A(x) B(x)$ in absolute value. We apply Fujiwara's result to obtain such a bound. We have that $A(x)=x^{5}+a_{4} x^{4}+a_{3} x^{3}+a_{2} x^{2}+a_{1} x+a_{0}$ and $B(x)=-x^{5}+b_{4} x^{4}+$ 
$b_{3} x^{3}+b_{2} x^{2}+b_{1} x+b_{0}$. Let $t=\max \{|a|,|b|,|c|\}$. First we compute bounds for the absolute value of the coefficients of $A(x)$ and $B(x)$. These are as follows

$$
\begin{aligned}
& \left|a_{4}\right| \leq 3 t^{2}+14 t+59 / 3, \\
& \left|a_{3}\right| \leq 16 / 9 t^{3}+28 t^{2}+392 / 3 t+3331 / 27, \\
& \left|a_{2}\right| \leq 29 / 9 t^{4}+112 / 3 t^{3}+392 / 3 t^{2}+2744 / 9 t+274, \\
& \left|a_{1}\right| \leq 16 / 9 t^{5}+70 / 3 t^{4}+392 / 3 t^{3}+2744 / 9 t^{2}+120, \\
& \left|a_{0}\right| \leq t^{6}+14 t^{5}+196 / 3 t^{4}+2744 / 27 t^{3}
\end{aligned}
$$

and

$$
\begin{aligned}
& \left|b_{4}\right| \leq 3 t^{2}+16 t+1 / 3, \\
& \left|b_{3}\right| \leq 16 / 9 t^{3}+32 t^{2}+512 / 3 t+1979 / 27, \\
& \left|b_{2}\right| \leq 29 / 9 t^{4}+128 / 3 t^{3}+512 / 3 t^{2}+4096 / 9 t+274, \\
& \left|b_{1}\right| \leq 16 / 9 t^{5}+80 / 3 t^{4}+512 / 3 t^{3}+4096 / 9 t^{2}+120, \\
& \left|b_{0}\right| \leq t^{6}+16 t^{5}+256 / 3 t^{4}+4096 / 27 t^{3} .
\end{aligned}
$$

One needs to establish a bound for $\left|a_{5-i}\right|^{1 / i}$ and $\left|b_{5-i}\right|^{1 / i}, i=1,2, \ldots, 5$. One has that $\max \left\{\left|a_{5-i}\right|^{1 / i},\left|b_{5-i}\right|^{1 / i}\right\} \leq 3 t^{2}+34 t$. Thus Fujiwara's bound implies that $|x| \leq 6 t^{2}+68 t$

\section{Proof of Theorem 3}

\section{Consider the Diophantine equation}

$$
\frac{x(x+1)(x+2)(x+3)(x+4)(x+5)}{(x+a)(x+b)(x+c)(x+d)}=y^{2},
$$

where $a, b, c, d \in \mathbb{Z}$ are pairwise distinct integers with $a, b, c, d \notin\{0,1,2,3,4,5\}$. The polynomial part of the Puiseux expansion of

$$
\left(\frac{x(x+1)(x+2)(x+3)(x+4)(x+5)}{(x+a)(x+b)(x+c)(x+d)}\right)^{1 / 2}
$$

is $P(x)=x+\frac{15-(a+b+c+d)}{2}$. Let

$$
\begin{aligned}
A(x)= & x(x+1)(x+2)(x+3)(x+4)(x+5) \\
& -(x+a)(x+b)(x+c)(x+d)\left(P(x)-\frac{1}{2}\right)^{2}
\end{aligned}
$$

and

$$
\begin{aligned}
B(x)= & x(x+1)(x+2)(x+3)(x+4)(x+5) \\
& -(x+a)(x+b)(x+c)(x+d)\left(P(x)+\frac{1}{2}\right)^{2} .
\end{aligned}
$$


The degree of $A(x)$ is 5 and the leading coefficient is 1 , the degree of $B(x)$ is also 5 and the leading coefficient is -1 . So one has that

$$
\frac{A(x)}{(x+a)(x+b)(x+c)(x+d)} \quad \text { and } \quad \frac{B(x)}{(x+a)(x+b)(x+c)(x+d)}
$$

have opposite signs if $|x|$ is larger than the maximum of the zeroes of $A(x) B(x)$ in absolute value. It follows that either

$$
\left(P(x)-\frac{1}{2}\right)^{2}<\frac{x(x+1)(x+2)(x+3)(x+4)(x+5)}{(x+a)(x+b)(x+c)(x+d)}<\left(P(x)+\frac{1}{2}\right)^{2}
$$

or

$$
\left(P(x)+\frac{1}{2}\right)^{2}<\frac{x(x+1)(x+2)(x+3)(x+4)(x+5)}{(x+a)(x+b)(x+c)(x+d)}<\left(P(x)-\frac{1}{2}\right)^{2} .
$$

We conclude that if $|x|$ is large, then $y=P(x)=x+\frac{15-(a+b+c+d)}{2}$ and $x$ divides the constant term of the polynomial

$$
4 x(x+1)(x+2)(x+3)(x+4)(x+5)-4(x+a)(x+b)(x+c)(x+d) P(x)^{2} .
$$

That is

$$
x \mid(a+b+c+d-15)^{2} a b c d .
$$

Now we compute bounds for $\left|a_{i}\right|$ and $\left|b_{i}\right|, i=0,1, \ldots, 4$, where $A(x)=x^{5}+$ $a_{4} x^{4}+a_{3} x^{3}+a_{2} x^{2}+a_{1} x+a_{0}$ and $B(x)=-x^{5}+b_{4} x^{4}+b_{3} x^{3}+b_{2} x^{2}+b_{1} x+b_{0}$. Let $t=\max \{|a|,|b|,|c|,|d|\}$. We have that

$$
\begin{aligned}
& \left|a_{4}\right| \leq 6 t^{2}+28 t+36 \\
& \left|a_{3}\right| \leq 6 t^{3}+28 t^{2}+196 t+225 \\
& \left|a_{2}\right| \leq 9 t^{4}+112 t^{3}+294 t^{2}+274, \\
& \left|a_{1}\right| \leq 12 t^{5}+98 t^{4}+196 t^{3}+120, \\
& \left|a_{0}\right| \leq 4 t^{6}+28 t^{5}+49 t^{4}
\end{aligned}
$$

and

$$
\begin{aligned}
& \left|b_{4}\right| \leq 6 t^{2}+32 t+21 \\
& \left|b_{3}\right| \leq 6 t^{3}+32 t^{2}+256 t+225 \\
& \left|b_{2}\right| \leq 9 t^{4}+128 t^{3}+384 t^{2}+274 \\
& \left|b_{1}\right| \leq 12 t^{5}+112 t^{4}+256 t^{3}+120 \\
& \left|b_{0}\right| \leq 4 t^{6}+32 t^{5}+64 t^{4}
\end{aligned}
$$

One obtains that $\max \left\{\left|a_{5-i}\right|^{1 / i},\left|b_{5-i}\right|^{1 / i}\right\} \leq 6 t^{2}+66 t$. Thus Fujiwara's bound implies that $|x| \leq 12 t^{2}+132 t$. 


\section{Numerical Results}

In what follows a solution $(x, y) \in \mathbb{Z}^{2}$ is called trivial if $y=0$. In this section we provide complete lists of non-trivial solutions of the considered three Diophantine equations for certain values of the parameters.

Theorem 5.1. Let $a, b \in\{-10,-9, \ldots, 14,15\} \backslash\{0,1,2,3,4,5\}$ with $a<$ $b$. The pairs $(a, b)$ for which equation (2.1) has a non-trivial solution are given by

\begin{tabular}{|c|c|}
\hline$(a, b)$ & list of non-trivial solutions $(x, y)$ \\
\hline \hline$(-10,-8)$ & {$[(3,24)]$} \\
\hline$(-10,-6)$ & {$[(1,4)]$} \\
\hline$(-9,-7)$ & {$[(2,12)]$} \\
\hline$(-9,-6)$ & {$[(-6,2)]$} \\
\hline$(-7,-3)$ & {$[(-7,6)]$} \\
\hline$(-6,-5)$ & {$[(1,6)]$,} \\
\hline$(-6,-2)$ & {$[(1,12),(-8,12)]$} \\
\hline$(-4,-2)$ & {$[(-10,30),(-6,3)]$} \\
\hline$(-4,7)$ & {$[(-10,60)]$} \\
\hline$(-2,9)$ & {$[(5,60)]$} \\
\hline$(7,9)$ & {$[(1,3),(5,30)]$} \\
\hline$(7,11)$ & {$[(12),(-6,12)]$} \\
\hline$(8,12)$ & {$[(2,6)]$} \\
\hline$(10,11)$ & {$[(-6,6)]$} \\
\hline$(11,14)$ & {$[(1,2)]$} \\
\hline$(11,15)$ & {$[(-6,4)]$} \\
\hline$(12,14)$ & {$[(-7,12)]$} \\
\hline$(13,15)$ & {$[(-8,24)]$} \\
\hline
\end{tabular}

Theorem 5.2. Let $a, b, c \in\{-7,-6, \ldots, 12\} \backslash\{0,1,2,3,4,5\}$ with $a<$ $b<c$. The triples $(a, b, c)$ for which equation (3.1) has a non-trivial solution are given by

\begin{tabular}{|c|c|}
\hline$(a, b, c)$ & list of non-trivial solutions $(x, y)$ \\
\hline \hline$(-7,-6,-4)$ & {$[(1,-2),(-8,-2)]$} \\
\hline$(-7,-5,-1)$ & {$[(-9,-3)]$} \\
\hline$(-7,-2,12)$ & {$[(-7,2)]$} \\
\hline$(-7,7,12)$ & {$[(2,-2)]$} \\
\hline$(-7,9,11)$ & {$[(1,-1)]$} \\
\hline$(-6,-4,12)$ & {$[(-6,1)]$} \\
\hline$(-6,-3,8)$ & {$[(1,2)]$} \\
\hline$(-6,6,10)$ & {$[(4,-6)]$} \\
\hline$(-5,-1,7)$ & {$[(-9,-6)]$} \\
\hline$(-5,-1,11)$ & {$[(-9,6)]$} \\
\hline$(-4,-3,-2)$ & {$[(-6,-1)]$} \\
\hline$(-4,-3,7)$ & {$[(-6,2)]$} \\
\hline$(-3,8,11)$ & {$[(-6,-2)]$} \\
\hline$(-2,6,10)$ & {$[(4,6)]$} \\
\hline$(-2,8,9)$ & {$[(1,-2)]$} \\
\hline$(6,10,12)$ & {$[(4,3)]$} \\
\hline$(7,8,9)$ & {$[(1,1)]$} \\
\hline$(9,11,12)$ & {$[(3,2),(-6,2)]$} \\
\hline
\end{tabular}

TheOREM 5.3. Let $a, b, c, d \in\{-7,-6, \ldots, 12\} \backslash\{0,1,2,3,4,5\}$ with $a<$ $b<c<d$. The tuples $(a, b, c, d)$ for which equation (4.1) has a non-trivial solution are given by 


\begin{tabular}{|c|c|}
\hline$(a, b, c, d)$ & list of non-trivial solutions $(x, y)$ \\
\hline$(-7,-6,-5,7)$ & {$[(-9,3)]$} \\
\hline$(-7,-6,-4,-3)$ & {$[(1,2)]$} \\
\hline$(-7,-6,-4,6)$ & $(-8,2)$ \\
\hline$(-7,-6,10,11)$ & {$[(-8,4)$} \\
\hline$(-7,-5,-1,6)$ & {$[(-9,3)$} \\
\hline$(-7,-5,6,10)$ & {$[(4,12)]$} \\
\hline$(-7,-4,-1,12)$ & {$[(2,6)]$} \\
\hline$(-7,-4,7,11)$ & {$[(3,6)]$} \\
\hline$(-7,-4,7,12)$ & {$[(2,2)]$} \\
\hline$(-7,-3,-2,6)$ & {$[(-7,2)$} \\
\hline$(-7,-3,6,12)$ & {$[(2,3)]$} \\
\hline$(-7,-3,8,11)$ & {$[(-7,3)]$} \\
\hline$(-7,-2,9,11)$ & {$[(1,1)]$} \\
\hline$(-7,-2,9,12)$ & {$[(-7,2)]$} \\
\hline$(-7,-1,8,12)$ & {$[(-7,3)]$} \\
\hline$(-7,6,9,12)$ & $(-7,6)$ \\
\hline$(-6,-5,7,8)$ & {$[(-9,12)]$} \\
\hline$(-6,-5,10,11)$ & {$[(4,12),(-9,12)]$} \\
\hline$(-6,-5,11,12)$ & {$[(3,4)]$} \\
\hline$(-6,-4,7,9)$ & {$[(-10,15)]$} \\
\hline$(-6,-4,7,12)$ & {$[(-6,1)]$} \\
\hline$(-6,-4,8,9)$ & $(-6,1)$ \\
\hline$(-6,-3,7,8)$ & {$[(1,1)]$} \\
\hline$(-6,-3,8,12)$ & {$[(2,3)]$} \\
\hline$(-6,-2,-1,7)$ & {$[(-8,4)]$} \\
\hline$(-6,-2,9,12)$ & {$[(-8,6)]$} \\
\hline$(-5,-3,-1,8)$ & {$[(-9,6)]$} \\
\hline$(-5,-3,8,9)$ & {$[(1,1)]$} \\
\hline$(-5,-1,6,8)$ & $\overline{[(-9,12)}$ \\
\hline$(-5,-1,10,12)$ & $(-9,12)$ \\
\hline$(-4,-3,7,8)$ & $(-6,2)$ \\
\hline$(-4,-3,8,10)$ & {$[(-6,1)$} \\
\hline$(-4,-3,9,11)$ & {$[(1,1)]$} \\
\hline$(-4,-2,-1,9)$ & $[5,30)]$ \\
\hline$(-4,-2,6,9)$ & $(-10,15)]$ \\
\hline$(-4,-2,9,11)$ & {$[(5,15)]$} \\
\hline$(-4,-1,7,9)$ & {$[(5,15)]$} \\
\hline$(-4,6,7,9)$ & {$[(-10,30)]$} \\
\hline$(-3,-2,8,9)$ & {$[(1,2)]$} \\
\hline$(-3,-2,8,11)$ & $(-6,1)]$ \\
\hline$(-3,-2,10,11)$ & $\overline{[(4,12)]}$ \\
\hline$(-3,-1,6,10)$ & {$[(4,12)]$} \\
\hline$(-3,6,8,10)$ & {$[(4,6)]$} \\
\hline$(-2,6,7,11)$ & {$[(3,4)]$} \\
\hline$(-2,10,11,12)$ & {$[(4,3)]$} \\
\hline$(-1,6,10,12)$ & {$[(4,3)]$} \\
\hline$(-1,7,8,12)$ & {$[(2,2)$} \\
\hline$(-1,9,11,12)$ & {$[(3,2)]$} \\
\hline$(8,9,11,12)$ & {$[(-6,2)]$} \\
\hline
\end{tabular}

Proofs of Theorems 4, 5 And 6. We wrote Sage ([22]) codes to compute all solutions $(x, y) \in \mathbb{Z}^{2}$ of the concrete equations. These can be downloaded from

http://shrek. unideb.hu/ tengely/RatFunErdosGraham. sage.

In the procedures approximate values of the real roots of the polynomials $A(x)$ and $B(x)$ are used instead of the bounds provided by Fujiwara's result. In this way we obtain better bounds for the "small" solutions. The "large" 
solutions are roots of certain polynomials with integral coefficients, therefore we factor these polynomials to determine these integral roots.

\section{REFERENCES}

[1] M. Bauer and M. A. Bennett, Applications of the hypergeometric method to the generalized Ramanujan-Nagell equation, Ramanujan J. 6 (2002), 209-270.

[2] M. A. Bennett, N. Bruin, K. Győry, and L. Hajdu, Powers from products of consecutive terms in arithmetic progression, Proc. London Math. Soc. 92 (2006), 273-306.

[3] M. A. Bennett and R. Van Luijk, Squares from blocks of consecutive integers: a problem of Erdös and Graham, Indag. Math., New Ser. 23 (2012), 123-127.

[4] L.E. Dickson, History of the theory of numbers. Vol II: Diophantine analysis, Chelsea Publishing Co., New York, 1966.

[5] P. Erdős, Note on the product of consecutive integers (II), J. London Math. Soc. 14 (1939), 245-249.

[6] P. Erdős and R. L. Graham, Old and new problems and results in combinatorial number theory, Monographies de L'Enseignement Mathématique, Geneva, 1980.

[7] P. Erdös and J. L. Selfridge The product of consecutive integers is never a power, Illinois J. Math. 19 (1975), 292-301.

[8] M. Fujiwara, Über die obere Schranke des absoluten Betrages der Wurzeln einer algebraischen Gleichung, Tôhoku Math. J. 10 (1916), 167-171.

[9] K. Györy, On the diophantine equation $n(n+1) \ldots(n+k-1)=b x^{\ell}$, Acta Arith. 83 (1998), 87-92.

[10] K. Győry, L. Hajdu, and Á. Pintér, Perfect powers from products of consecutive terms in arithmetic progression, Compos. Math. 145 (2009), 845-864.

[11] K. Györy, L. Hajdu, and N. Saradha, On the Diophantine equation $n(n+d) \cdots(n+$ $(k-1) d)=b y^{l}$, Canad. Math. Bull. 47 (2004), 373-388.

[12] L. Hajdu, Sz. Tengely, and R. Tijdeman, Cubes in products of terms in arithmetic progression, Publ. Math. Debrecen 74 (2009), 215-232.

[13] N. Hirata-Kohno, S. Laishram, T. N. Shorey, and R. Tijdeman, An extension of a theorem of Euler, Acta Arith. 129 (2007), 71-102.

[14] S. Laishram and T. N. Shorey, The equation $n(n+d) \cdots(n+(k-1) d)=b^{2}$ with $\omega(d) \leq 6$ or $d \leq 10^{10}$, Acta Arith. 129 (2007), 249-305.

[15] F. Luca and P.G. Walsh, On a diophantine equation related to a conjecture of Erdös and Graham, Glas. Mat. Ser. III 42 (2007), 281-289.

[16] R. Obláth, Über das Produkt fünf aufeinander folgender Zahlen in einer arithmetischen Reihe, Publ. Math. Debrecen 1 (1950), 222-226.

[17] O. Rigge, Über ein diophantisches problem in: 9th Congress Math. Scand., Helsingfors 1938., 155-160.

[18] A. Sankaranarayanan and N. Saradha, Estimates for the solutions of certain Diophantine equations by Runge's method, Int. J. Number Theory 4 (2008), 475-493.

[19] N. Saradha, On perfect powers in products with terms from arithmetic progressions, Acta Arith. 82 (1997), 147-172.

[20] N. Saradha and T. N. Shorey, Almost squares in arithmetic progression, Compositio Math. 138 (2003), 73-111.

[21] M. Skałba, Products of disjoint blocks of consecutive integers which are powers, Colloq. Math. 98 (2003), 1-3.

[22] W. A. Stein et al., Sage Mathematics Software (Version 6.0), The Sage Development Team, 2014, http://www.sagemath.org. 
[23] Sz. Tengely, Note on the paper: "An extension of a theorem of Euler" [Acta Arith. 129 (2007), 71-102; MR2326488] by N. Hirata-Kohno, S. Laishram, T. N. Shorey and R. Tijdeman. Acta Arith. 134 (2008), 329-335.

[24] Sz. Tengely and N. Varga, On a generalization of a problem of Erdös and Graham, Publ. Math. Debrecen 84 (2014), 475-482.

[25] M. Ulas, On products of disjoint blocks of consecutive integers, Enseign. Math. (2) 51 (2005), 331-334.

Sz. Tengely

Mathematical Institute

University of Derecen

P.O.Box 12, 4010 Debrecen

Hungary

E-mail: tengely@science.unideb.hu

N. Varga

Institute of Mathematics

MTA-DE Research Group "Equations, Functions and Curves"

Hungarian Academy of Sciences and University of Debrecen

P. O. Box 12, H-4010 Debrecen

Hungary

E-mail: nvarga@science.unideb.hu

Received: 10.2.2014. 